\title{
Heavy-quark mesons in the diabatic approach: string breaking and the quarkoniumlike spectrum
}

\author{
Roberto Bruschini ${ }^{1, *}$ \\ ${ }^{1}$ Unidad Teórica, Instituto de Física Corpuscular (Universidad de Valencia-CSIC), E-46980 Paterna \\ (Valencia), Spain
}

\begin{abstract}
The Born-Oppenheimer approximation provides a description of heavy-quark mesons firmly based on lattice QCD, but its validity is limited to the lightest states lying far below the first open-flavour meson-meson threshold. This limitation can be overcome in the diabatic framework, a formalism first introduced in molecular physics, where the dynamics is encoded in a potential matrix whose elements can be derived from unquenched lattice QCD studies of string breaking. The off-diagonal elements of the potential matrix provide interaction between heavy quark-antiquark and meson-meson pairs, from which the mixing of quarkonium states with molecular components and the OZI-allowed strong decay widths are directly calculated. This allows for a QCD-based unified description of conventional quarkonium and unconventional mesons containing quark-antiquark and meson-meson components, what has proved to be successful for charmoniumlike and bottomoniumlike resonances.
\end{abstract}

\section{Introduction}

For many years the spectrum of heavy-quark mesons has been successfully described by the quark model, in its nonrelativistic [1,2] and semi-relativistic [3] realizations, from the bound states of a heavy quark-antiquark $(Q \bar{Q})$ pair. This is until the discovery of $X(3872)$ [4], which ushered heavy-quark meson spectroscopy in a new era. In fact this state, and many others discovered afterwards [5], have mass and decay properties that clearly defy the quark model predictions. Finding a unified description of the conventional and unconventional states from QCD, the theory of strong interactions, has been a long standing problem in heavy-quark meson spectroscopy ever since.

A general feature of the unconventional heavy-quark meson states is that their mass lies close to some open-flavor meson-meson threshold with the same quantum numbers. This fact seems to indicate that a proper description of them must at least incorporate meson-meson configurations on top of the quark-antiquark component. Actually, the effect of open flavor meson-meson thresholds on the quarkonium spectrum has been extensively studied through phenomenological models such as the Cornell or ${ }^{3} P_{0}$ models [1, 2, 6-18], however these approaches lack a direct connection with QCD.

A connection with QCD can be found using the Born-Oppenheimer (B-O) approximation [19-21]. More precisely, because the mass of the heavy quarks is much larger than the QCD

\footnotetext{
*e-mail: roberto.bruschini@ific.uv.es
} 
energy scale associated to the gluon field, heavy-quark meson states can be associated to solutions of a Schrödinger-like equation with effective potentials. These potentials correspond to the energy levels of a stationary gluon field in presence of static $Q$ and $\bar{Q}$ sources, and can be calculated directly in lattice QCD. In this way conventional quarkonium $Q \bar{Q}$ states can be identified with the solutions of the ground state (Cornell) potential, and quarkonium hybrids $Q \bar{Q} g$ (where $g$ stands for a gluon) are described through the solutions of the excited state potentials.

However, it is customary to neglect configuration mixing when applying the B-O approximation in QCD, which limits its validity when light quark flavors are included in the calculation of the static potentials [21]. Concretely, lattice QCD calculations of the energy levels of stationary gluon and light quark fields in presence of static $Q \bar{Q}$ sources have shown that as the energy of a quarkonium $Q \bar{Q}$ configuration approaches the mass of an open-flavor meson-meson pair a sizable mixing between the two of them (string breaking) takes place $[22,23]$. Therefore, the validity of the B-O approximation without configuration mixing is limited to states whose mass lies far below any open-flavor meson-meson threshold.

In this talk we shall overcome this limitation using the diabatic approach, first developed in molecular physics [24], to obtain a QCD-based description of heavy-quark mesons taking into account the configuration mixing between $Q \bar{Q}$ and meson-meson. In this approach the $Q \bar{Q}$-meson-meson mixing induced by string breaking is incorporated together with the confining $Q-\bar{Q}$ interaction in a potential matrix that is directly connected to the static energy levels calculated in lattice QCD. The solution of the multichannel Schrödinger equation with this potential matrix gives the spectrum of both conventional (containing mainly $Q \bar{Q}$ ) and unconventional (with significant meson-meson component) heavy-quark meson states as well as their OZI-allowed decay widths. This framework is then applied to study charmoniumlike $[25,26]$ and bottomoniumlike [27] resonances.

The contents of the talk are organized as follows. In Sec. 2 we recap the main steps in the derivation of the B-O approximation. In Sec. 3 we introduce the diabatic approach in QCD and use it to explore the $Q \bar{Q}-$ meson-meson mixing. The results of a diabatic study of the charmoniumlike and bottomoniumlike spectra are recollected in Sec. 4, and the main conclusions are summarized in Sec. 5.

\section{Born-Oppenheimer approximation}

The B-O approximation, first developed for the description of molecules [28], is particularly useful to describe physical systems where there is a clear separation between "heavy" and "light" degrees of freedom (d.o.f.). In molecules this division is provided by the electrons being several thousands times lighter than the nuclei, while in heavy-quark meson the distinction is provided by the QCD energy scale $\Lambda_{\mathrm{QCD}}$ associated to the gluon fields. So heavy quark flavors such as charm $(c)$ and bottom $(b)$, whose mass is much bigger than $\Lambda_{\mathrm{QCD}}$, are going to be treated as "heavy" d.o.f. whereas gluons $(g)$ and light quark flavors up $(u)$, down $(d)$, and strange $(s)$ are going to be treated as "light" fields.

The huge separation of the energy scales of the light and heavy fields is crucial to the assumption underlying the B-O approximation: that the evolution of the heavy d.o.f. is so slow with respect to that of the light fields that the dynamics of the latter can be solved for static heavy quark sources. So in the B-O approximation a heavy-quark meson state $|\psi\rangle$ (containing both heavy and light fields) is a solution of the characteristic equation

$$
H|\psi\rangle=E|\psi\rangle
$$

with $E$ the energy of the state and $H$ the Hamiltonian

$$
H=K_{Q \bar{Q}}+H_{Q \bar{Q}}^{\mathrm{lf}}
$$


where $K_{Q \bar{Q}}$ is the kinetic energy of the heavy quark-antiquark pair and $H_{Q \bar{Q}}^{\mathrm{lf}}$ a Hamiltonian operator for the light fields that in general depends on the $Q \bar{Q}$ coordinates but does not contain any derivative with respect to them.

The first step in the solution of (1) is to solve the dynamics of the light fields for static heavy quarks. Formally, this corresponds to putting $K_{Q \bar{Q}}$ to zero, so that the coordinates of the $Q \bar{Q}$ cease being a dynamical variable and become instead a parameter of the residual Hamiltonian for the light fields: $H_{Q \bar{Q}}^{\mathrm{lf}} \rightarrow H_{\text {static }}^{\mathrm{lf}}(r)$ where $r$ is the relative $Q \bar{Q}$ distance. The solution of the secular equation for $H_{\text {static }}^{\text {lf }}(r)$,

$$
H_{\text {static }}^{\mathrm{lf}}(r)\left|\zeta_{i}(r)\right\rangle=V_{i}(r)\left|\zeta_{i}(r)\right\rangle
$$

with $i=0,1, \ldots$ labeling the ground and excited state solutions, yields the light fields states $\left|\zeta_{i}(r)\right\rangle$ and the corresponding static energy levels $V_{i}(r)$, which can be calculated in lattice QCD.

After having solved the light fields for static heavy quarks, the motion of the heavy d.o.f. is reintroduced using the so-called adiabatic expansion of the heavy-quark meson state

$$
|\psi\rangle=\sum_{i} \int \mathrm{d} r \psi_{i}(r)|r\rangle\left|\zeta_{i}(r)\right\rangle
$$

where $|r\rangle$ is the quantum state indicating the $Q \bar{Q}$ relative position $r$ and $\psi_{i}(r)$, the coefficients of the expansion, are called the adiabatic wave functions of the heavy-quark meson state. It is important to highlight that the light-field states $\left|\zeta_{i}(r)\right\rangle$ used in the adiabatic expansion (4) are calculated at the same position $r$ of the heavy quarks, what makes it the most natural expansion in the idealized situation where the light fields states adjust instantaneously to the motion of the $Q \bar{Q}$ (hence the name).

Plugging the adiabatic expansion back into (1), and after some manipulations, one can see that the evolution of the adiabatic wave functions $\psi_{i}(r)$ is determined by the Schrödinger-like equation

$$
\sum_{j}\left[-\frac{\hbar^{2}}{2 \mu_{Q \bar{Q}}}\left(\delta_{i j} \nabla+\tau_{i j}(r)\right)^{2}+\left(V_{i}(r)-E\right) \delta_{i j}\right] \psi_{i}(r)=0,
$$

where the adiabatic potentials $V_{i}(r)$ are nothing but the static energy levels calculable in lattice QCD. One should realize though that Eq. (5) is not a proper Schrödinger equation because of the non-adiabatic coupling terms $\tau(r)_{i j}$ appearing in the kinetic energy term. These terms, defined as $\tau(r)_{i j} \equiv\left\langle\zeta_{i}(r) \mid \nabla \zeta_{j}(r)\right\rangle$, reflect the general nontrivial dependence of the light-field states on the motion of the heavy d.o.f. and couple the various adiabatic wave functions to each other. In B-O it is customary to neglect these terms, what corresponds to the so-called single-channel approximation. Notice that this approximation can be deemed valid only if no significant mixing between the light-field states takes place.

The adiabatic potentials have been first calculated in quenched lattice QCD, this is, without including light quark flavors in the calculation of the energy levels (see for example $[19,20])$. They are represented in Fig. 1 . The ground state potential shows an attractive Coulomb behavior at short distances and a linear confining shape at large distances, corresponding to the quarkonium $Q \bar{Q}$ configuration. The first excited state on the other hand, while still being confining at large distances, is repulsive at small distances, and is usually associated to the first quarkonium hybrid $Q \bar{Q} g$ configuration.

Thus the B-O approximation can be used to obtain a description of quarkonium and quarkonium hybrid states that is firmly based on quenched lattice QCD, but what happens if light quark flavors are introduced among the light fields? The adiabatic potentials recently 


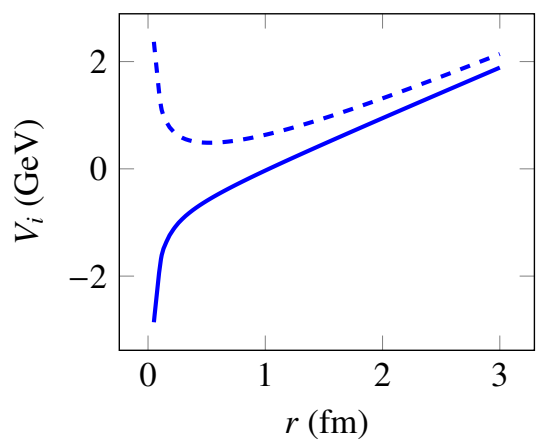

Figure 1. Representation of the adiabatic potentials in quenched lattice QCD. Solid line stands for the ground state (quarkonium) potential and dashed line for the first excited (hybrid) potential.

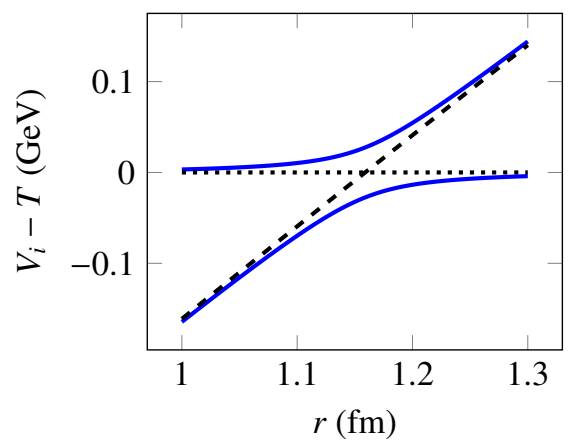

Figure 2. Representetion of the adiabatic potentials in unquenched lattice QCD "normalized" with respect to the threshold mass $T$. Solid lines stand for the ground state and first excited potentials, dashed line for the quarkonium potential, and dotted line for the threshold energy.

calculated in unquenched (with gluons and light quarks) lattice QCD [22, 23], and represented in Fig. 2, show that the single-channel approximation is untenable when open-flavor meson-meson configurations start to play some role. In fact one can see in Fig. 2 that as $r$ increases the energy of the ground state shifts progressively from linearly increasing, corresponding to the confining $Q \bar{Q}$ potential, to the constant threshold mass value, corresponding to a noninteracting open-flavor meson-meson configuration. At the same time an excited state potential showing the opposite behavior appears above threshold. Notice that these two potentials, unlike the pure $Q \bar{Q}$ and meson-meson energies also represented in Fig. 2, do not cross each other. This phenomenon, referred to as avoided crossing, reflects the creation of a light quark-antiquark pair from the sea by string breaking and their recombination with the heavy quark-antiquark to create an open-flavor meson-meson pair. This configuration mixing between $Q \bar{Q}$ and meson-meson clearly breaks the single-channel approximation and therefore limits its validity to the energy region far below threshold.

\section{Diabatic approach}

To solve the complications that arise in the B-O approximation when dealing with string breaking, we use a more convenient expansion for the heavy-quark meson state $|\psi\rangle$ : the 
diabatic expansion

$$
|\psi\rangle=\sum_{i} \int \mathrm{d} r \widetilde{\psi}_{i}\left(r, r_{0}\right)|r\rangle\left|\zeta_{i}\left(r_{0}\right)\right\rangle,
$$

whose crucial difference with respect to the adiabatic expansion (4) is that the light-field states are now calculated for a fixed distance $r_{0}$ instead of the $Q \bar{Q}$ distance $r$. So if we choose $r_{0}$ far from the avoided crossing then the expansion coefficients, the diabatic wave functions $\widetilde{\psi}_{i}\left(r, r_{0}\right)$, correspond to either pure $Q \bar{Q}$ or meson-meson: $\widetilde{\psi}_{i}\left(r, r_{0}\right) \rightarrow \psi_{Q \bar{Q}}(r), \psi_{M \bar{M}}(r)$. In other words, in the diabatic framework the state $|\psi\rangle$ is expanded in $Q \bar{Q}$ and meson-meson components, instead of adiabatic channels that correspond to a mixing of both.

With the diabatic expansion, Eq. (1) becomes the diabatic Schrödinger equation

$$
\left[\left(\begin{array}{cc}
-\frac{\nabla^{2}}{2 \mu_{Q \bar{Q}}} & 0 \\
0 & -\frac{\nabla^{2}}{2 \mu_{M \bar{M}}}
\end{array}\right)+\left(\begin{array}{cc}
V_{Q \bar{Q}}(r) & V_{\text {mix }}(r) \\
V_{\text {mix }}(r) & T_{M \bar{M}}
\end{array}\right)-E\right]\left(\begin{array}{l}
\psi_{Q \bar{Q}}(r) \\
\psi_{M \bar{M}}(r)
\end{array}\right)=0,
$$

where $\mu_{Q \bar{Q}}$ and $\mu_{M \bar{M}}$ are respectively the $Q \bar{Q}$ and meson-meson reduced masses, $V_{Q \bar{Q}}(r)$ is the $Q \bar{Q}$ potential, $T_{M \bar{M}}$ the open-flavor meson-meson threshold mass, and $V_{\text {mix }}(r)$ the $Q \bar{Q}-$ meson-meson mixing potential. Unlike its counterpart Eq. (5), this is a standard multichannel Schrödinger equation with a diabatic potential matrix coupling the two channels through the off-diagonal element $V_{\text {mix }}(r)$.

The diabatic potential matrix enjoys a direct connection with the energy levels calculated in lattice QCD, in fact, its eigenvalues are nothing but the unquenched adiabatic potentials [24]. Using this correspondence and the calculated energy levels it can be shown that near the avoided crossing the mixing potential $V_{\text {mix }}(r)$ must be proportional to the energy gap between the adiabatic potentials, whereas far from the avoided crossing its modulus must decrease quite rapidly [25]. The simplest parametrization that respects this requirements is a Gaussian one

$$
\left|V_{\text {mix }}(r)\right|=\frac{\Delta}{2} e^{-\frac{\left(V_{Q \bar{Q}}(r)-T_{M \bar{M}}\right)^{2}}{2 \Lambda^{2}}}
$$

where $\Delta$ is the energy gap and $\Lambda$ an energy scale regulating the size of the region where the mixing takes place. Notice that the correspondence between the eigenvalues of the diabatic potential matrix and the adiabatic potentials fixates only the modulus of the mixing potential, not its sign.

The solution of the diabatic Schrödinger equation (7) gives a unified description of heavyquark mesons composed in general of $Q \bar{Q}$ and open-flavor meson-meson components that is firmly based on lattice QCD, both above and below threshold. Comparison of the resulting spectrum with that of bare quarkonium states shows that the mixing has multiple effects. In general, heavy-quark meson states above threshold acquire decay widths to open-flavor meson-meson pairs, and their mass is shifted due to the coupling with the continuum of meson-meson states. Below threshold, the coupling with off-shell meson-meson configurations makes that all heavy-quark meson states possess some molecular component, and some unconventional state (usually with a significant molecular component) may appear near some threshold.

\section{Results}

We shall now recollect the results of a diabatic study of the bottomoniumlike [27] and chamroniumlike $[25,26]$ spectra. Let us notice that the parameters we use in the diabatic potential matrix are not actually fitted on lattice data but are instead a mixture of lattice and phenomenology. There are two reasons for this. First, unquenched lattice QCD energy levels 


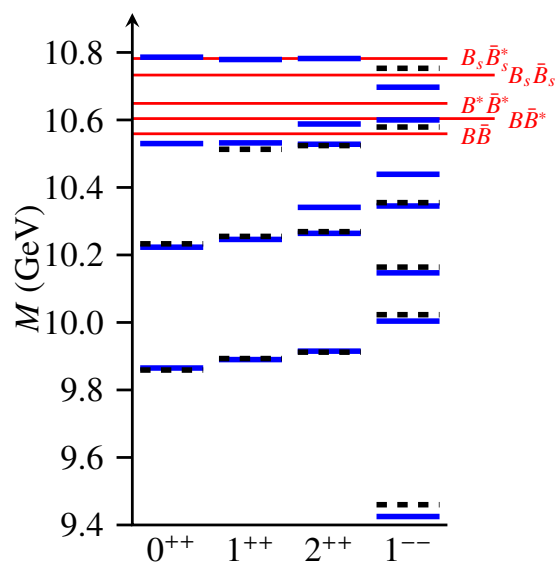

(a) Bottomoniumlike spectrum.

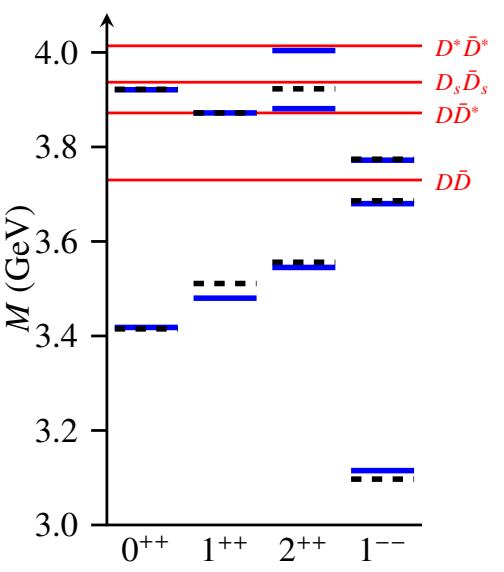

(b) Charmoniumlike spectrum.

Figure 3. Spectrum of bottomoniumlike (left) and charmoniumlike (right) meson states. Thick solid lines stand for states calculated in the diabatic approach, thick dashed lines stand for well-established experimental candidates, and long thin horizontal lines stand for open-flavor meson-meson thresholds.

Table 1. Calculated OZI-allowed strong decay widths of bottomoniumlike states above threshold. When available, the experimental total width $\Gamma_{\text {total }}^{\text {Expt }}$ of the corresponding experimental candidate is reported. Masses and widths in $\mathrm{MeV}$ units.

\begin{tabular}{rrrrrrrr}
\hline$J^{P C}$ & $M$ & $\Gamma_{B \bar{B}}$ & $\Gamma_{B \bar{B}^{*}}$ & $\Gamma_{B^{*} \bar{B}^{*}}$ & $\Gamma_{B_{s} \bar{B}_{s}}$ & $\Gamma_{\text {total }}^{\text {Theor }}$ & $\Gamma_{\text {total }}^{\text {Expt }}$ \\
\hline $0^{++}$ & 10785.8 & 1.6 & & 5.3 & 0.7 & 7.6 & \\
$1^{++}$ & 10778.9 & & 0.2 & 1.7 & & 1.9 & \\
$2^{++}$ & 10588.4 & 4.3 & & & & 4.3 & \\
$2^{++}$ & 10782.3 & 5.4 & 1.5 & 21.0 & 10.4 & 38.3 & \\
$1^{--}$ & 10599.8 & 21.9 & & & & 21.9 & $20.5 \pm 2.5$ \\
$1^{--}$ & 10697.0 & 2.0 & 1.0 & 38.0 & & 41.0 & \\
\hline
\end{tabular}

are only currently available for bottomonium and not for charmonium. Second, a successful description of the low-lying conventional quarkonium states is essential for the description of the unconventional ones, as it depends crucially on the position of the underlying $Q \bar{Q}$ bound states with respect to the open-flavor meson-meson thresholds. Hence, the parameters of the $Q \bar{Q}$ potential are taken from phenomenology, the threshold values are taken as the sum of the corresponding experimental meson masses, and the parameters of the mixing potential are taken from lattice calculations in the bottomoniumlike case, or fitted from the mass of $X(3872)$ in the charmoniumlike case. Notice also that the results presented here have little or no dependence on the particular choice of the parametrization for the mixing potential (8), as long as it respects the conditions of its modulus being approximately maximum near the avoided crossing and vanishing away from it.

The calculated spectra are illustrated in Fig. 3, while the calculated OZI-allowed strong decay widths of quarkoniumlike states above threshold are reported in Tables 1 and 2. From them we can see that the diabatic approach can give a reasonable and unified account of the 
Table 2. Calculated OZI-allowed strong decay widths of charmoniumlike states above threshold. When available, the experimental total width $\Gamma_{\text {total }}^{\text {Expt }}$ of the corresponding experimental candidate is reported. Masses and widths in $\mathrm{MeV}$ units.

\begin{tabular}{rrrrrrr}
\hline$J^{P C}$ & $M$ & $\Gamma_{D \bar{D}}$ & $\Gamma_{D \bar{D}^{*}}$ & $\Gamma_{D_{s} \bar{D}_{s}}$ & $\Gamma_{\text {total }}^{\text {Theor }}$ & $\Gamma_{\text {total }}^{\text {Expt }}$ \\
\hline $0^{++}$ & 3920.9 & 0.6 & & & 0.6 & \\
$2^{++}$ & 3881.1 & 49.5 & 0.4 & & 49.9 & $35.3 \pm 2.8$ \\
$2^{++}$ & 4003.9 & 4.8 & 6.3 & 3.5 & 14.5 & \\
$1^{--}$ & 3771.7 & 20.2 & & & 20.2 & $27.2 \pm 1.0$ \\
\hline
\end{tabular}

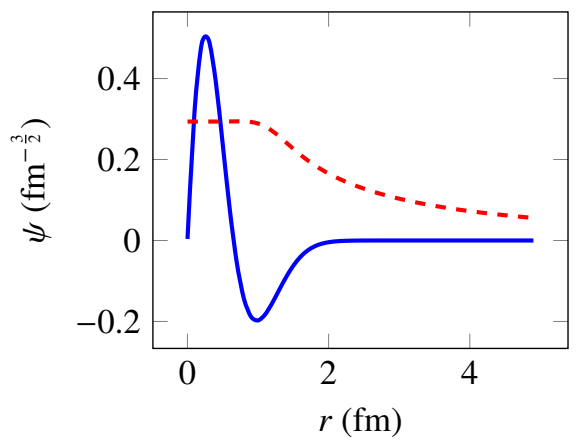

Figure 4. Diabatic wave function of $X(3872)$. The solid and dashed lines stand for the $c \bar{c}$ and $D \bar{D}^{*}$ components respectively.

spectrum of conventional and unconventional quarkoniumlike states. We see that this is composed of conventional quarkonium states plus a certain number of unconventional ones lying close to some open-flavor meson-meson threshold. The fact that the predicted widths are fairly close to the few well-established experimental values (within a $10 \%$ and $30 \%$ accuracy for bottomoniumlike and charmoniumlike states respectively) gives further support to the mixing potential being the main mechanism driving both the appearance of unconventional quarkoniumlike states and the OZI-allowed decay of states above threshold.

It may be interesting to examine in more detail the description of $X(3872)$ that emerges in the diabatic approach. From Fig. 4 it can be seen that the diabatic $X(3872)$ at short distance is made of both $c \bar{c}$ and $D \bar{D}^{*}$ components in comparable amounts. Then as the distance increases the confined $c \bar{c}$ component vanishes and the long $D \bar{D}^{*}$ tail dominates. This makes the $X(3872)$ mostly a $D \bar{D}^{*}$ molecule $\left(97 \%\right.$ total $D \bar{D}^{*}$ probability vs. $\left.3 \% c \bar{c}\right)$, with a calculated mean root-mean-square radius of near $11 \mathrm{fm}$.

\section{Summary}

To summarize, the Born-Oppenheimer approximation provides a description of conventional quarkonia and quarkonium hybrids that is firmly based on lattice QCD. This description takes the energy levels calculated in quenched lattice QCD as the potentials of a Schrödinger equation whose solution provides the quarkoniumlike meson spectrum and wave functions. The diabatic approach allows to include open-flavor meson-meson components in this description. In this approach the dynamics is governed by a diabatic potential matrix whose eigenvalues 
are directly connected to the unquenched lattice QCD static potentials. This completely nonperturbative description treats the quark-antiquark-meson-meson mixing from string breaking on the same grounds as the confining quark-antiquark interaction. The diabatic approach has been applied to study bottomoniumlike and charmoniumlike mesons, showing that it can give account of both the spectrum and OZI-allowed decay widths of conventional as well as unconventional states.

This work has been supported by Ministerio de Ciencia e Innovación and Agencia Estatal de Investigación of Spain and European Regional Development Fund Grant PID2019-105439 GB-C21 and by EU Horizon 2020 Grant No. 824093 (STRONG-2020). The author acknowledges a FPI fellowship from Ministerio de Ciencia, Innovación y Universidades of Spain under Grant No. BES-2017-079860.

\section{References}

[1] E. Eichten, K. Gottfried, T. Kinoshita, K.D. Lane, T.M. Yan, Phys. Rev. D 17, 3090 (1978), [Erratum: Phys. Rev. D 21, 313 (1980)]

[2] E.J. Eichten, K. Lane, C. Quigg, Phys. Rev. D 69, 094019 (2004)

[3] S. Godfrey, N. Isgur, Phys. Rev. D 32, 189 (1985)

[4] S.K. Choi et al. (Belle Collaboration), Phys. Rev. Lett. 91, 262001 (2003)

[5] P.A. Zyla et al. (Particle Data Group), Prog. Theor. Exp. Phys. 2020, 083 C01 (2020)

[6] E.J. Eichten, K. Lane, C. Quigg, Phys. Rev. D 73, 014014 (2006), [Erratum: Phys. Rev. D 73, 079903 (2006)]

[7] E. van Beveren, G. Rupp, T.A. Rijken, C. Dullemond, Phys. Rev. D 27, 1527 (1983)

[8] E. van Beveren, G. Rupp, Prog. Part. Nucl. Phys. 117, 103845 (2021)

[9] K. Heikkilä, N.A. Törnqvist, S. Ono, Phys. Rev. D 29, 110 (1984)

[10] T. Barnes, S. Godfrey, E.S. Swanson, Phys. Rev. D 72, 054026 (2005)

[11] T. Barnes, E.S. Swanson, Phys. Rev. C 77, 055206 (2008)

[12] M.R. Pennington, D.J. Wilson, Phys. Rev. D 76, 077502 (2007)

[13] I.V. Danilkin, Y.A. Simonov, Phys. Rev. D 81, 074027 (2010)

[14] I.V. Danilkin, V.D. Orlovsky, Y.A. Simonov, Phys. Rev. D 85, 034012 (2012)

[15] J. Ferretti, G. Galatà, E. Santopinto, Phys. Rev. C 88, 015207 (2013)

[16] J. Ferretti, E. Santopinto, Phys. Rev. D 90, 094022 (2014)

[17] Z.Y. Zhou, Z. Xiao, Eur. Phys. J. A 50, 165 (2014)

[18] P.G. Ortega, J. Segovia, D.R. Entem, F. Fernández, Phys. Lett. B 778, 1 (2018)

[19] K.J. Juge, J. Kuti, C.J. Morningstar, Phys. Rev. Lett. 82, 4400 (1999)

[20] G.S. Bali, Phys. Rep. 343, 1 (2001)

[21] E. Braaten, C. Langmack, D.H. Smith, Phys. Rev. D 90, 014044 (2014)

[22] G.S. Bali, H. Neff, T. Düssel, T. Lippert, K. Schilling (SESAM Collaboration), Phys. Rev. D 71, 114513 (2005)

[23] J. Bulava, B. Hörz, F. Knechtli, V. Koch, G. Moir, C. Morningstar, M. Peardon, Phys. Lett. B 793, 493 (2019)

[24] M. Baer, Beyond Born-Oppenheimer: Electronic Nonadiabatic Coupling Terms and Conical Intersections (John Wiley \& Sons, New York, 2006)

[25] R. Bruschini, P. González, Phys. Rev. D 102, 074002 (2020)

[26] R. Bruschini, P. González, Phys. Rev. D 103, 074009 (2021)

[27] R. Bruschini, P. González, Phys. Rev. D 103, 114016 (2021)

[28] M. Born, R. Oppenheimer, Ann. Phys. (Berlin) 389, 457 (1927) 